\title{
Descritores quantitativos na estimativa da divergência genética entre genótipos de mamoneira utilizando análises multivariadas ${ }^{1}$
}

\author{
Quantitative descriptors to estimative genetic divergence in castor bean genotypes \\ based on multivariate analysis
}

\author{
Francisco Valdevino Bezerra Neto ${ }^{2 *}$, Nilton Rocha Leal ${ }^{3}$, Leandro Simões Azeredo Gonçalves ${ }^{3}$, Luiz de Morais \\ Rêgo Filho ${ }^{4}$ e Antonio Teixeira do Amaral Júnior ${ }^{3}$
}

\begin{abstract}
Resumo - O conhecimento da diversidade é de fundamental importância para o melhoramento de plantas, pois além de identificar combinações que possam expressar elevada heterose, aumenta a perspectiva de seleção de segregantes superiores, com a potencialização da variabilidade no cruzamento entre genótipos divergentes. Diversos métodos multivariados podem ser aplicados no estudo da diversidade e a escolha baseia-se na precisão desejada pelo pesquisador, na facilidade da análise e na forma como os dados foram obtidos. A presente pesquisa teve como objetivos avaliar a diversidade de onze genótipos de mamoneira (Ricinus communis L.) nas regiões Norte e Noroeste do Estado do Rio de Janeiro e investigar o potencial do uso dos genótipos avaliados em futuros programas de melhoramento. Foram avaliados seis descritores quantitativos (altura de planta, comprimento total dos racemos, comprimento da região com frutificação do racemo, comprimento da região sem frutificação no racemo, número de frutos por racemo e produção total), utilizando-se delineamento de blocos ao acaso com quatro repetições. Houve diferenças significativas entre os tratamentos para todas as características avaliadas. As análises multivariadas (UPGMA, Tocher e Variáveis Canônicas) foram similares formando três grupos. Os genótipos Nordestina, IAC 80, Al Guarany, Paraguaçu, Mirante e IAC 226 revelaram-se promissores para as regiões avaliadas. Em programas de intercruzamentos, recomendam-se as combinações Al Guarany x Nordestina, Al Guarany x IAC 80 e Nordestina x IAC 80.
\end{abstract}

Palavras-chave - Ricinus communis L. Análise multivariada. Genética vegetal. Plantas-melhoramento genético.

\begin{abstract}
Knowledge of the diversity is of fundamental importance for plant breeding, as well as for the identification of combinations that can express high heterosis levels, increase the expectation of selection of superior segregating genotypes, based on the accumulation of varibility by crosses between dissimilar genotypes. Several multivariate methods can be used to investigate diversity. The choice is made according to the accuracy desired by the researcher, the ease of the analysis and how the data were obtained. The purpose of this study was to evaluate the diversity of 11 castor bean genotypes in the north and northwest of Rio de Janeiro, Brazil and to investigate the potential of each genotype for future breeding programs. Six quantitative traits (plant height, total raceme length, length of the region with fruiting raceme, length of the region without fruiting raceme, number of fruits per raceme and total yield) were evaluated in a randomized complete block design with four replications. The differences between treatments were significant for all traits evaluated. Results of multivariate analyses (UPGMA, Tocher and canonical variables) showed similar results forming three groups each. The genotypes Nordestina, IAC 80, Al Guarany, Paraguaçu, Mirante and IAC 226 proved promising for the studied regions. For crossing or breeding programs, the combinations Al Guarany x Nordestina, Al Guarany x IAC 80 and Nordestina x IAC 80 are recommended.
\end{abstract}

Key words - Ricinus communis L. Multivariate analysis. Plant genetics. Plant breeding.

\footnotetext{
* Autor para correspondência

'Recebido para publicação em 17/07/2009; aprovado em 23/04/2010

Parte da Tese de Doutorado do primeiro autor apresentada ao Programa de Pós-Graduação em Genética e Melhoramento de Plantas/UENF ${ }^{2}$ Instituto Federal do Espírio Santo, Campus Itapina, Rodovia BR-259, km 70, Colatina-ES, Brasil, 29709-910, francisco.neto@ifes.edu.br

${ }^{3}$ Universidade Estadual do Norte Fluminense Darcy Ribeiro/UENF, Av. Alberto Lamego n. 2000, Campos dos Goytacazes-RJ, Brasil, 28013-602, nilton@uenf.br, lsagrural@yahoo.com.br, amaraljr@uenf.br

${ }^{4}$ Empresa de Pesquisa Agropecuária do Estado do Rio de Janeiro/PESAGRO-RIO, Av. Francisco Lamego n. 134, Campos dos Goytacazes-RJ, Brasil, 28000-000,1morais@pesagro.rj.gov.br
} 


\section{Introdução}

A mamoneira (Ricinus communis L.) é nativa da África tropical e atualmente, é cultivada em regiões tropicais e subtropicais do mundo (ALLAN et al., 2008). O óleo da mamona possui alto valor econômico em razão da sua versatilidade de aplicação na indústria, podendo ser utilizado na composição de cosméticos, de produtos farmacêuticos, de óleos lubrificantes, além de polímeros (ALLAN et al., 2008; SEVERINO et al., 2009; TAKANO et al., 2007). A versatilidade do óleo de mamona se deve à estrutura química doácido ricinoléico(INTERNATIONAL CASTOR OIL ASSOCIATION - ICOA, 2008).

Entre os maiores produtores, a Índia detém $68 \%$ da produção mundial, seguida por China e Brasil, com 18 e $8 \%$, respectivamente $(\mathrm{FAO}, 2008)$. No Brasil, a produção está concentrada principalmente no semi-árido e no Estado da Bahia, com mais de $80 \%$ da produção e da área plantada (CARVALHO, 2005). No que se refere à produtividade média, o Brasil, com estimativa de $587,00 \mathrm{~kg} \mathrm{ha}^{-1}$, situa-se abaixo da Índia e da China, cujas produtividades médias são de 1.064,00 e 954,00 kg.ha-1 ${ }^{-1}$, respectivamente (FAO, 2008).

A baixa produtividade nacional deve-se, principalmente, à utilização de sementes de baixo potencial genético, bem como de cultivares não adaptadas às regiões de cultivo (COSTA et al., 2006). Neste contexto, instituições como a Embrapa e outros centros de pesquisa desenvolvem programas de melhoramento genético, visando principalmente aumentar a produtividade (CAVALCANTE et al., 2008; HALL et al., 2009; SEVERINO et al., 2006).

Nos programas de melhoramento de plantas, informações relacionadas com a diversidade são de fundamental importância, pois permitem identificar combinações hibridas que possam produzir altos efeitos heteróticos, além de proporcionar maior variabilidade genética nas gerações segregantes (BAHIA et al., 2008; GONÇALVES et al., 2009; ROCHA et al., 2009). Vários métodos multivariados podem ser usados na predição da diversidade genética. A escolha baseia-se na precisão desejada pelo pesquisador, bem como na facilidade da análise e na forma como os dados foram obtidos.

Entre os métodos multivariados, destacamse a análise por componentes principais, as variáveis canônicas e os métodos de agrupamentos hierárquicos e de otimização (CRUZ et al., 2004; GONÇALVES et al., 2008; SUDRÉ et al., 2007; SUDRÉ et al., 2010). Trabalhos recentes foram desenvolvidos visando à determinação da diversidade genética da mamona (ALLAN et al., 2008; BAHIA et al., 2008; CAVALCANTE et al., 2008; COSTA et al., 2006; FIGUEIREDO NETO et al., 2004; SEVERINO et al., 2009).
Nas regiões Norte e Noroeste do Estado do Rio de Janeiro, nas quais predomina a agricultura familiar, a mamoneira torna-se uma alternativa viável de renda para as pequenas propriedades e para a fixação de trabalhadores na área rural. A presente pesquisa foi desenvolvida visando quantificar a diversidade genética entre onze genótipos de mamoneira cultivados nas regiões Norte e Noroeste Fluminense, bem como indicar combinações híbridas para futuros trabalhos de melhoramento genético com a espécie.

\section{Material e métodos}

Foram avaliados onze genótipos de mamoneira, sendo dois híbridos (Lyra e Savana) e nove cultivares (IAC-80, Al Guarany, Paraguaçu, Nordestina, Mirante, Nativa, Cafelista, Tito e IAC 226). Os genótipos foram semeados no primeiro semestre de 2008, na Estação Experimental de Campos dos Goytacazes, pertencente à Empresa de Pesquisa Agropecuária do Estado do Rio de Janeiro (PESAGRO-RIO).

Foram avaliadas seis características: (a) altura de planta, em metros (ALT); (b) comprimento total dos racemos, em cm (CR); (c) comprimento da região com frutificação do racemo, em cm (CRF); (d) comprimento da região sem frutificação no racemo, em cm (CSF); (e) número de frutos por racemo, em unidades (NFR) e (f) produção total, em kg ha-1 (PROD). Utilizou-se o delineamento de blocos ao acaso com quatro repetições, sendo cada parcela formada por três linhas de sete metros de comprimento, com espaçamento entre linhas de dois metros, e entre plantas de um metro, considerando como útil a linha central onde cinco plantas foram consideradas.

Inicialmente, os dados foram submetidos à análise de variância para averiguação de variabilidade genética entre os acessos e, em seguida, os valores médios foram ordenados segundo o teste de agrupamento Scott-Knott (SCOTT; KNOTT, 1974), a 5\% de probabilidade.

As análises multivariadas foram implementadas por meio de técnicas de agrupamento hierárquico, com base nos métodos UPGMA, Ward e Vizinho Mais Próximo, utilizando a distância generalizada de Mahalanobis como medida de dissimilaridade (CRUZ et al., 2004; CRUZ, 2008; MOHAMMADI; PRASANNA, 2003;). A otimização foi verificada por meio do método de Tocher (CRUZ et al., 2004; CRUZ, 2008), bem como pelo procedimento das Variáveis Canônicas (CRUZ et al., 2004).

Para testar a eficiência de cada método de agrupamento hierárquico estimou-se o coeficiente de correlação cofenética (CCC). Posteriormente, calculou-se 
o número de grupos ideal utilizando os critérios pseudo-F e pseudo-t² (GONÇALVES et al., 2008; MINGOTI, 2007).

Os dados foram analisados utilizando-se os recursos computacionais dos programas GENES (CRUZ, 2008), R (R DEVELOPMENT CORE TEAM, 2006) e SAS (SAS INSTITUTE, 1990).

\section{Resultados e discussão}

$\mathrm{Na}$ análise de variância univariada, todas as características expressaram diferenças significativas entre os genótipos. Isto indica que os materiais vegetais estudados são agronomicamente diferentes, o que sugere a necessidade de avaliação das médias separadamente de cada genótipo e seu agrupamento pelo procedimento de Scott e Knott (SCOTT; KNOTT, 1974) (Tabela 1).

No agrupamento dos genótipos, as características comprimento total do racemo (CR) e comprimento da região sem frutificação no racemo (CSF) apresentaram as maiores variabilidades, com a formação de quatro grupos, seguidas por comprimento da região com frutificação do racemo (CRF) e número de frutos por racemo (NFR), em que três grupos foram constituídos. A altura da planta (ALT) e a produção total (PROD) compuseram apenas dois grupos. A análise lógica do agrupamento das médias genotípicas permite identificar a ocorrência de variabilidade entre os materiais estudados, o que já foi indicado no teste F. Este fato tem importância para o aproveitamento da heterose e na obtenção de segregantes com constituições genotípicas de interesse para o Norte e Noroeste Fluminense em programas de melhoramento genético com mamoneira.

Os materiais Nordestina, Al Guarany, IAC 80, Paraguaçu, Mirante e IAC 226 tiveram produtividades acima de $1.000 \mathrm{~kg} \mathrm{ha}^{-1}$. Em relação ao comprimento total dos racemos (CR), o genótipo IAC 80 apresentou o maior valor, seguido por Al Guarany, Cafelista e IAC 226, o que consequenciou em maior número de frutos por racemo para esses genótipos (Tabela 1).

$\mathrm{Na}$ análise fundamentada por Variáveis Canônicas (CAN), verificou-se que as duas primeiras variáveis explicaram 92,94\% da variância total acumulada, sendo CAN1 responsável por $82,59 \%$ e CAN2 por $10,35 \%$ (Figura 1). Estes valores são considerados satisfatórios para representação gráfica bi-dimensional, o que é possível quando as Variáveis Canônicas explicam mais de $80 \%$ da variância contida no conjunto de características analisadas

Tabela 1 - Estimativas de médias ${ }^{1}$ aritméticas de onze genótipos de mamoneira para seis descritores agronômicos, agrupados pelo procedimento Scott-Knott. Campos dos Goytacazes, RJ, Brasil, 2008

\begin{tabular}{lcccrrr}
\hline \multirow{2}{*}{ Genótipos } & \multicolumn{5}{c}{ Características(1) } \\
\cline { 2 - 7 } IAC 80 & ALT & CTR & CFR & CSF & NFR & PROD \\
Al Guarany & $1,97 \mathrm{a}$ & $61,38 \mathrm{a}$ & $44,38 \mathrm{a}$ & $16,99 \mathrm{~b}$ & $101,93 \mathrm{a}$ & $1.405,00 \mathrm{a}$ \\
Paraguaçu & $1,61 \mathrm{~b}$ & $51,25 \mathrm{~b}$ & $26,48 \mathrm{~b}$ & $24,77 \mathrm{a}$ & $83,80 \mathrm{~b}$ & $1.535,75 \mathrm{a}$ \\
Nordestina & $2,16 \mathrm{a}$ & $22,19 \mathrm{~d}$ & $13,71 \mathrm{c}$ & $8,47 \mathrm{~d}$ & $32,18 \mathrm{c}$ & $1.205,00 \mathrm{a}$ \\
Savana & $2,46 \mathrm{a}$ & $40,00 \mathrm{c}$ & $26,24 \mathrm{~b}$ & $13,76 \mathrm{c}$ & $59,85 \mathrm{c}$ & $1.617,50 \mathrm{a}$ \\
Lyra & $1,08 \mathrm{~b}$ & $30,63 \mathrm{~d}$ & $24,69 \mathrm{~b}$ & $5,94 \mathrm{~d}$ & $56,78 \mathrm{c}$ & $960,00 \mathrm{~b}$ \\
Mirante & $1,33 \mathrm{~b}$ & $25,96 \mathrm{~d}$ & $16,31 \mathrm{c}$ & $9,65 \mathrm{~d}$ & $45,93 \mathrm{c}$ & $758,13 \mathrm{~b}$ \\
IAC 226 & $2,17 \mathrm{a}$ & $35,75 \mathrm{c}$ & $27,07 \mathrm{~b}$ & $8,68 \mathrm{~d}$ & $57,95 \mathrm{c}$ & $1.120,00 \mathrm{a}$ \\
Cafelista & $2,03 \mathrm{a}$ & $48,33 \mathrm{~b}$ & $28,68 \mathrm{~b}$ & $19,65 \mathrm{~b}$ & $107,6 \mathrm{a}$ & $1.046,67 \mathrm{a}$ \\
Nativa & $1,42 \mathrm{~b}$ & $49,94 \mathrm{~b}$ & $31,27 \mathrm{~b}$ & $18,67 \mathrm{~b}$ & $73,59 \mathrm{~b}$ & $619,38 \mathrm{~b}$ \\
Tito & $2,56 \mathrm{a}$ & $28,19 \mathrm{~d}$ & $16,38 \mathrm{c}$ & $11,81 \mathrm{c}$ & $33,88 \mathrm{c}$ & $610,00 \mathrm{~b}$ \\
\hline Média & $1,88 \mathrm{a}$ & $25,20 \mathrm{~d}$ & $16,48 \mathrm{c}$ & $8,72 \mathrm{~d}$ & $44,73 \mathrm{c}$ & $450,00 \mathrm{~b}$ \\
\hline QM Trat ${ }^{(2)}$ & 1,87 & 38,07 & 24,69 & 13,37 & 63,47 & $1.029,77$ \\
\hline CVe & $0,88^{(3)}$ & $675,76^{* *}$ & $314,15^{* *}$ & $139,93^{* *}$ & $2617,61 * *$ & $616318,15^{* *}$ \\
\hline
\end{tabular}

(1)ALT: altura da planta (m); CTR: comprimento total dos racemos (cm); CRF: comprimento da região com frutificação do racemo (cm); CSF: comprimento da região sem frutificação no racemo $(\mathrm{cm})$; NFR: número de frutos por racemo (unid); PROD: Produção total (Kg.ha ${ }^{-1}$ ); médias com letras iguais não diferem entre si, pelo teste de Scott \& Knott, a 5\% de probabilidade. ${ }^{(2)}$ QM Trat: Quadrado Médio do Tratamento; e **: Significativo no nível de $1 \%$ de probabilidade pelo teste $\mathrm{F} ;{ }^{(3)} \mathrm{CVe}$ : Coeficiente de variação experimental 
(CRUZ et al., 2004). Pela análise visual do gráfico bidimensional, pode-se inferir que três grupos foram formados, sendo que o grupo I reuniu apenas o genótipo Al Guarany; o grupo II, os genótipos IAC 226, Cafelista, Lyra, Nordestina, Tito, Paraguaçu e Nativa; e o grupo III, com os genótipos IAC 80, Savana e Mirante (Figura 1).

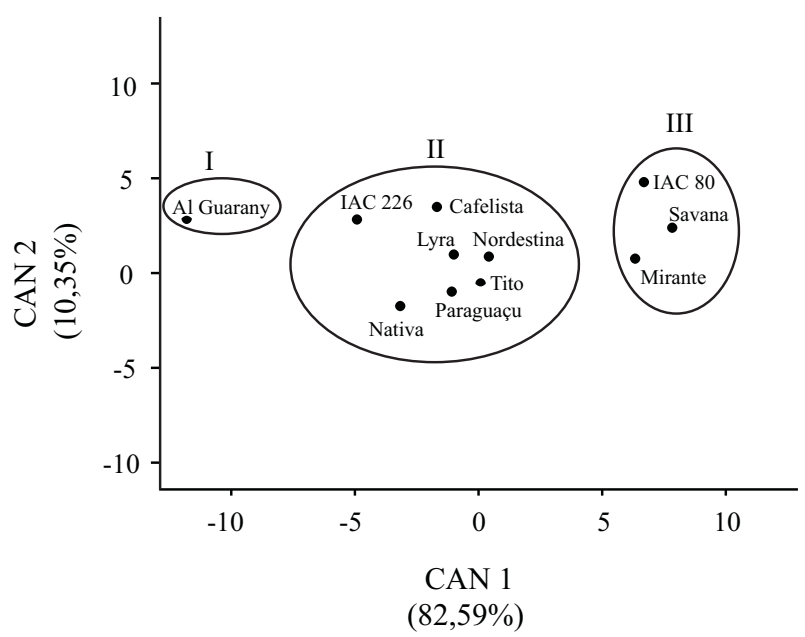

Figura 1 - Dispersão gráfica dos escores em relação aos eixos representativos das variáveis canônicas (CAN1 e CAN2) relativos a seis características avaliadas em onze genótipos de mamoneira. Campos dos Goytacazes, RJ, Brasil, 2008

A aplicação do método de otimização via Tocher permitiu identificar a formação de três grupos, de forma análoga aos grupos formados pelas Variáveis Canônicas (Tabela 2). Na análise de agrupamento hierárquico, o método UPGMA mostrou maior correlação cofenética quando comparado com os métodos Ward e Vizinho Mais Próximo, com valores respectivos de 0,76;0,60 e 0,72. Na concepção de Sokal e Rohlf (1962) valores abaixo de 0,80 indicam baixa representatividade das reais distâncias entre os genótipos por meio da representação gráfica do dendrograma, tornando-se não confiável.

Tabela 2 - Agrupamento de otimização entre onze genótipos ${ }^{1 /}$ de mamoneira, obtido pelo método Tocher, com base em seis características, utilizando-se a distância generalizada de Mahalanobis. Campos dos Goytacazes, RJ, Brasil, 2008

\begin{tabular}{|c|c|}
\hline Grupos & Genótipos $^{(1)}$ \\
\hline I & $\begin{array}{lllllll}3 & 4 & 6 & 8 & 9 & 10 & 11\end{array}$ \\
\hline II & 157 \\
\hline III & 2 \\
\hline
\end{tabular}

(1) 1: IAC 80; 2: Al Guarany; 3: Paraguaçu; 4: Nordestina; 5: Savana; 6: Lyra; 7: Mirante; 8: IAC 226; 9: Cafelista; 10: Nativa; 11: Tito
O critério do pseudo-F não permitiu a determinação do número ótimo de grupos, pois o F calculado foi monotonicamente crescente com o número de grupos formados, denotando a inexistência de uma partição natural dos dados. Contudo, pelo critério pseudo- $\mathrm{t}^{2}$ pode-se observar que o ponto máximo $(94,1)$ foi alcançado na formação de três grupos. Segundo Mingoti (2007), o fundamento dos critérios pseudo-F e pseudo- $\mathrm{t}^{2}$ está relacionado com o teste de hipótese, ou seja, é como se em cada passo do processo de agrupamento estivesse sendo feito um teste para comparação dos vetores de médias dos dois grupos que se uniram para formar um novo grupo. Por conseguinte, interessam os maiores valores de pseudo-F e pseudo- $\mathrm{t}^{2}$, vez que estariam relacionados com a menor probabilidade de significância do teste e, dessa forma, estaria rejeitando a igualdade de vetores de médias com maior significância. Nesse sentido, se a igualdade dos vetores de médias for rejeitada, os dois grupos não deveriam se unir para formar um novo grupo.

Pelos grupos formados por meio do agrupamento UPGMA utilizando-se o critério do pseudo- $\mathrm{t}^{2}$ (Figura 2), observou-se similaridade com a constituição dos grupos de Tocher e das Variáveis Canônicas. De acordo com Sokal e Rohlf (1962), o valor do coeficiente de correlação cofenético (CCC) para o agrupamento UPGMA seria não confiável, pois o mesmo expressou estimativa baixa de CCC, com a magnitude de 0,76 . Todavia, a similaridade de formação dos grupos por UPGMA com Tocher e Variáveis Canônicas, sobretudo com este último, por reter a quase totalidade da variação nos dois primeiros vetores $(92,94 \%)$, torna frágil a classificação proposta por Sokal e Rohlf (1962).

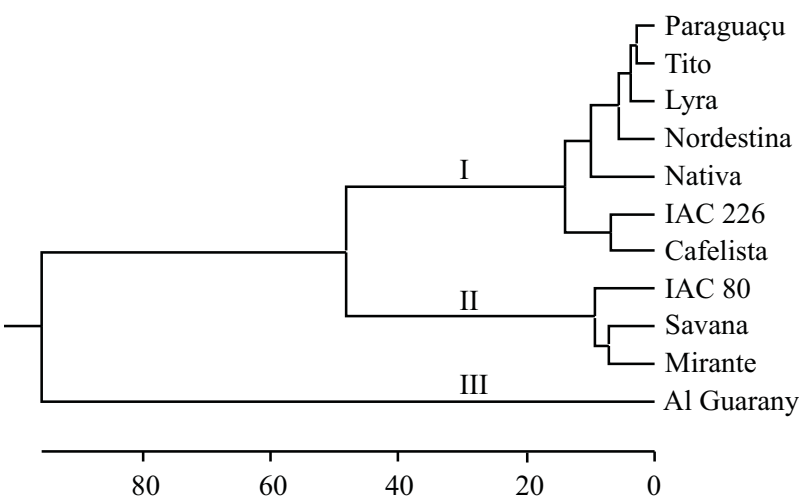

Figura 2 - Dendrograma de dissimilaridades genéticas entre onze genótipos de mamoneira, obtido pelo método UPGMA, com base em seis características quantitativas, utilizando-se a distância generalizada de Mahalanobis. Campos dos Goytacazes, RJ, Brasil, 2008 
Para os produtores do Norte e Noroeste do Estado do Rio de Janeiro, têm-se como promissores os genótipos Nordestina, IAC 80, Al Guarany, Paraguaçu, Mirante e IAC 226. Em relação, a constituição de futuros programas de melhoramento com mamoneira para as regiões Norte e Noroeste Fluminense, as combinações entre Al Guarany e Nordestina; Al Guarany e IAC 80; e Nordestina e IAC 80 tornam-se promissores, devido, estes genótipos apresentarem desempenhos superiores para as principais características de importância agronômicas e serem divergentes.

\section{Conclusões}

1. Houve elevada variabilidade genética entre os genótipos de mamoneira avaliados.

2. O critério pseudo- $\mathrm{t}^{2}$, ao proporcionar os mesmos grupos para UPGMA em comparação aos agrupamentos de Tocher e Variáveis Canônicas, coloca em xeque a classificação do coeficiente de correlação cofenética de Sokal e Rohlf (1962).

3. Os genótipos Nordestina, IAC 80, Al Guarany, Paraguaçu, Mirante e IAC 226 são promissores para o Norte e Noroeste Fluminense, com expectativa de superioridade das hibridações entre Al Guarany e Nordestina, Al Guarany e IAC 80, e Nordestina com IAC 80.

\section{Agradecimentos}

ÀPESAGRO-RIOeatodosaquelesquecontribuíram para que o artigo efetivamente fosse produzido.

\section{Referências}

ALLAN, G. et al. Worldwide genotyping of castor bean germplasm (Ricinus communis L.) using AFLPs and SSRs. Genetics Resources and Crop Evolution, v. 55, n. 03, p. 365-378, 2008

BAHIA, H. F. et al. Divergência genética entre cinco cultivares de mamoneira. Pesquisa Agropécuaria Brasileira, v. 43, n. 03, p. 357-362, 2008.

CARVALHO, B. C. L. Manual de cultivo da mamona. Salvador: EBDA, 2005. 65 p.

CAVALCANTE, M. et al. Divergência genética entre acessos de mamona em dez municípios de Alagoas. Revista Caatinga, v. 21, n. 03, p. 111-115, 2008.

COSTA, M. N. et al. Divergência genética entre acessos e cultivares de mamoneira por meio de estatística multivariada.
Pesquisa Agropecuária Brasileira, v. 41, n. 11, p. 16171622, 2006.

CRUZ, C. D. et al. Modelos biométricos aplicados ao melhoramento genético. Viçosa: UFV, 2004. 390 p.

CRUZ, C. D. Programa Genes diversidade genética. Viçosa: UFV, 2008. 278 p.

FOOD AND AGRICULTURE ORGANIZATION OF THE UNITED NATIONS (FAO). The agricultural production. 2008. Disponível em: <http: //www.faostat.fao.org $>$. Acesso em: 2 jan. 2009.

FIGUIEREDO NETO, A. et al. Divergência genética em acessos de mamona (Ricinus communis L.) baseada nas características de semente. Revista de Biologia e Ciência da Terra, v. 04, n. 02, p. 1-10, 2004.

GONÇALVES, L. S. A. et al. Comparison of multivariate statistical algorithms to cluster tomato heirloom accessions. Genetics and Molecular Research, v. 07, n. 04, p. 12891297, 2008.

GONÇALVES, L. S. A. et al. Heirloom tomato gene bank: assessing genetic divergence based on morphological, agronomic and molecular data using a Ward-modified location model. Genetics and Molecular Research, v. 08, n. 01, p. 364-374, 2009.

HALL, J. et al. Brazilian biofuels and social exclusion: established and concentrated ethanol versus emerging and dispersed biodiesel. Journal of Cleaner Production, v. 17, n. 01, p. 77-85, 2009.

INTERNATIONAL CASTOR OIL ASSOCIATION (ICOA). The chemistry of castor oil and its derivaties and their applications. 2008. Disponível em: $<$ http: //www.icoa.org/bull2. htm>. Acesso em: 20 dez. 2008.

MINGOTI, S. A. Análise de dados através de métodos de estatística multivariada: uma abordagem aplicada. Belo Horizonte: UFMG, 2007. 297 p.

MOHAMMADI S. A.; PRASANNA, B. M. Analysis of genetic diversity in crop plants - salient statistical tools and considerations. Crop Science, v. 43, n. 04, p. 1235-1248, 2003.

R FOUNDATION FOR STATISTICAL COMPUTING A language and environment for statistical computing. Vienna: $\mathrm{R}$ Foundation for Statistical Computing, 2006. Disponível $<$ http://www.r-project.org $>$. Acesso em: 13 mar. 2008.

ROCHA, M. C. et al. Descritores quantitativos na determinação da divergência genética entre acessos de tomateiro do grupo cereja. Ciência Rural, v. 39, n. 03, p. 664-670, 2009.

SAS INSTITUTE. SAS/STAT user's guide. Cary: Statistical Analysis System Institute. 1990. 956 p.

SCOTT, A. J.; KNOTT, M. A. Cluster analysis methods for grouping, means in the analysis of variance. Biometrics, v. 30, n. 03, p. $507-512,1974$.

SEVERINO, L. S. et al. Avaliação da produtividade e teor de óleo de dez genótipos de mamoneira cultivados em altitude 
inferior a 300 metros. Revista Ciência Agronômica, v. 37, n. 02, p. 188-194, 2006.

SEVERINO, L. S. et al. Variabilidade no percentual de tegumento da semente de mamona e sua importância para o melhoramento. Revista Ciência Agronômica, v. 40, n. 01, p. 94-98, 2009.

SOKAL, R. R.; ROHLF, F. J. The comparison of dendrograms by objective methods. Taxon, v. 11, n. 01, p. 30-40, 1962.

SUDRÉ, C. P. et al. Genetic resources of vegetable crops: a survey in the Brazilian germplasm collections pictured through papers published in the journals of the Brazilian Society for Horticultural Science. Horticultura Brasileira, v. 25, n. 04, p. 496-503, 2007.

SUDRÉ, C. P. et al. Genetic variability in domesticated Capsicum spp. as assessed by morphological and agronomic data in mixed statistical analysis. Genetics and Molecular Research, v. 09, n. 01, p. 283-294, 2010.

TAKANO, E. H. et al. Inibição do desenvolvimento de fungos fitopatogênicos por detergentes derivado de óleo da mamona (Ricinus communis). Ciência Rural, v. 37, n. 05, p. 1235-1240, 2007. 